University for Business and Technology in Kosovo

UBT Knowledge Center

UBT International Conference

2014 UBT International Conference

Nov 7th, 4:30 PM - 4:45 PM

\title{
Logistical Route Optimization to Reduce Transportation Cost
}

\author{
Bashkim Çerkini \\ KBRCompany, bashkimqerkini@gmail.com \\ Valma Prifti \\ Polytechnic University of Tirana, valmaprifti1@gmail.com \\ Robert Kosova \\ Aleksandër Moisiu University, robertko60@yahoo.com
}

Follow this and additional works at: https://knowledgecenter.ubt-uni.net/conference

Part of the Computer Sciences Commons

\section{Recommended Citation \\ Çerkini, Bashkim; Prifti, Valma; and Kosova, Robert, "Logistical Route Optimization to Reduce Transportation Cost" (2014). UBT International Conference. 55. \\ https://knowledgecenter.ubt-uni.net/conference/2014/all-events/55}

This Event is brought to you for free and open access by the Publication and Journals at UBT Knowledge Center. It has been accepted for inclusion in UBT International Conference by an authorized administrator of UBT Knowledge Center. For more information, please contact knowledge.center@ubt-uni.net. 


\title{
Logistical Route Optimization to Reduce Transportation Cost
}

\author{
Bashkim Çerkini ${ }^{1}$, Valma Prifti ${ }^{2}$, Robert Kosova ${ }^{3}$ \\ 1'KBR Company" Camp Bondsteel, Ferizaj, KOSOVË. \\ 2"Polytechnic University of Tirana", Tirana, ALBANIA. \\ 3“Aleksandër Moisiu" University, Durrës, ALBANIA. \\ bashkimqerkini@gmail.com ${ }^{1}$, valmaprifti1@gmail.com² , robertko60@yahoo.com $^{3}$
}

\begin{abstract}
Many manufacturing companies produce their products in few locations and transport them to the different shops or demand points.

In this paper work we will use the power of IP - Solver and Microsoft Solver Foundation in order to resolve logistical route optimization problem to reduce transportation cost of the fruit juice factory or to find the least expensive way to produce and transport products to the customers and meet customer demand. Fruit juice company produces juice in three different factories: F1, F2 and F3. Each week, the fruit juice factory must transport juice to the five different demand points: D1, D2, D3, D4 and D5. Speaking mathematical terms, our goal is to find minimal cost logistical route while passing all predetermined points on the way. This kind of problem is called:"A transportation problem" and can be set up as a integer linear programming model with the below specifications: Minimizing total production and shipping cost, Constraints - The amount shipped from each production point cannot exceed plant capacity. Every demand point must receive its required demand. Transportation trucks have the limit of loading quantity. Also, each shipping amount must be nonnegative

At the end we will compare IP - Solver and Microsoft Solver Foundation results and least expensive way with the utmost expensive way.
\end{abstract}

Keywords: Cost, Integer Programming, mathematical model, IP - Solver and Microsoft Solver Foundation.

\section{Introduction}

In this paper we choose a company that produces fruit juice in three different factories and shipping its products to the five different locations or demand points as shown in the figure below:

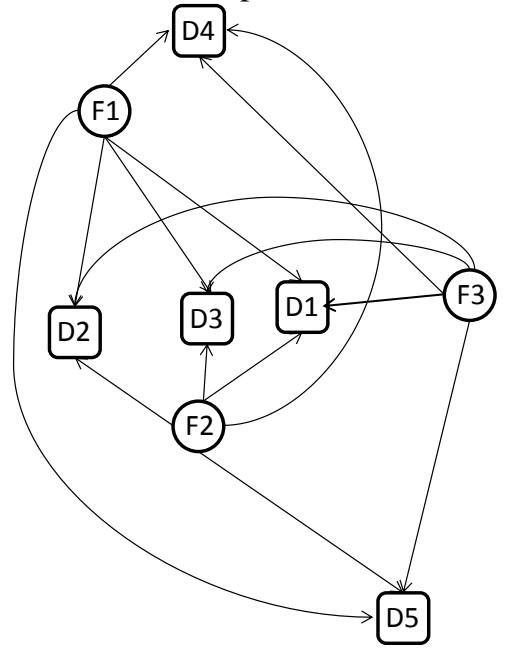

Figure 1. Distribution Network from Production Points to the Demands Points 


\section{Minimizing Cost for Fruit Juice Transportation}

Minimizing cost as optimization transportation problem is a typical problem in Supply Chain Optimization field. This problem is especially important in distribution and retail environments with transportation management needs. For example, many companies plan and do organize deliveries of goods from distribution centers to stores. Many times these deliveries consist of multiple segments. A likely question is: "What would be the most optimal route for the truck if it needs to pass number of points on its way?

In this walkthrough we will utilize the power of Microsoft Solver and Microsoft Solver Foundation in order to resolve Logistical route optimization problem. Technically speaking our objective is to find a least cost logistical route while passing all predefined points on the way.

Mathematical expression for calculation transportation cost per juice litter is:

$\mathbf{C}=(\mathbf{c k} * \mathbf{L}) / \mathbf{m}$

[1]

ck - Operator cost per $1 \mathrm{~km}$

$\mathbf{L}$ - Total length between production points and demand point

$\mathbf{m}$ - Is average capacity of truck loading with juice per juice litter

The total cost (C) is proportional to the length of the routes. Operator cost (ck) includes maintenance and direct costs of operation (driver wage, fuel consumption, tires, brake shoes, etc.) [4]. Vehicle depreciation costs are included as a part of operator cost too. Based on the mathematical expression [1] and data's we have the cost per litter shown in the table below:

Table 1. Cost per juice litter from production point to demand point

\begin{tabular}{|l|l|l|l|l|l|}
\hline Production Point & Shop 1 & Shop 2 & Shop 3 & Shop 4 Shop 5 \\
\hline Factory 1 & $\$ 0.10$ & $\$ 0.07$ & $\$ 0.08$ & $\$ 0.04$ & $\$ 0.09$ \\
\hline Factory 2 & $\$ 0.06$ & $\$ 0.05$ & $\$ 0.04$ & $\$ 0.10$ & $\$ 0.10$ \\
\hline Factory 3 & $\$ 0.05$ & $\$ 0.06$ & $\$ 0.07$ & $\$ 0.11$ & $\$ 0.16$ \\
\hline
\end{tabular}

The mathematical model (integer linear programming formulation) for this task will look like following:

$$
\operatorname{Min} \sum_{i}^{m} \sum_{j}^{n} C i j * S i j
$$

Constraints:

$$
\begin{array}{lr}
\sum_{j}^{n} S i j \leq F i & i=1, \ldots, \mathrm{m} \\
\sum_{i}^{m} S i j=D j & \mathrm{j}=1, \ldots, \mathrm{n} \\
5 \mathrm{ij} \leq \max \mathrm{T} & i=1, \ldots, \mathrm{m} \\
& \mathrm{j}=1, \ldots, \mathrm{n} \\
5 \mathrm{ij} \geq 0 & i=1, \ldots, \mathrm{m} \\
& \mathrm{j}=1, \ldots, \mathrm{n}
\end{array}
$$




\section{Minimizing Transportation Cost}

Minimizing Transportation Cost can be set up as a linear Solver model with the following specifications:

Target cell - Minimize total production and shipping cost.

Changing cells - The amount produced at each production point that is shipped to each shop.

Constraints - The amount shipped from each production point can't exceed plant capacity. Each demand point must receive at least its required demand. Also, every changing cell cannot be negative.

Below table shows first page of the Microsoft Excel Solver

Table 2. First page of the Microsoft Excel Solver

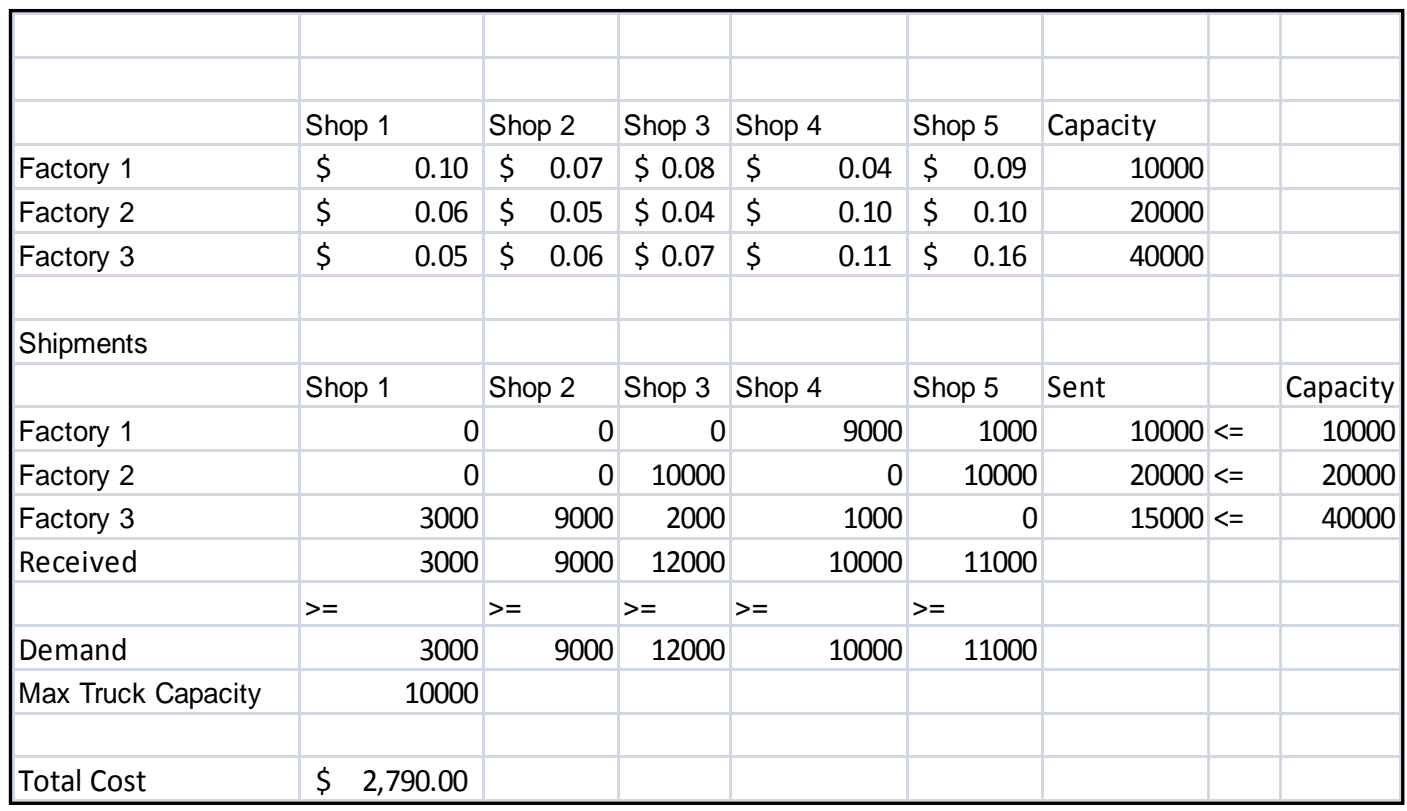

Cells B4:F6 contains cost from table 2. Cell B18 is Target Cell. Changing cells are B10:F12. Constraints (Sent) G10:G12 are less or equal to Capacity I10:I12. Constraints (Received) B13:F13 are bigger or equal to the demand B15:F15. Cells B15: F15 contains demands for each shop. I10:I12 is production capacity and BF16 is Maximum truck transportation capacity.

All above constrains are entered into Microsoft Solver.

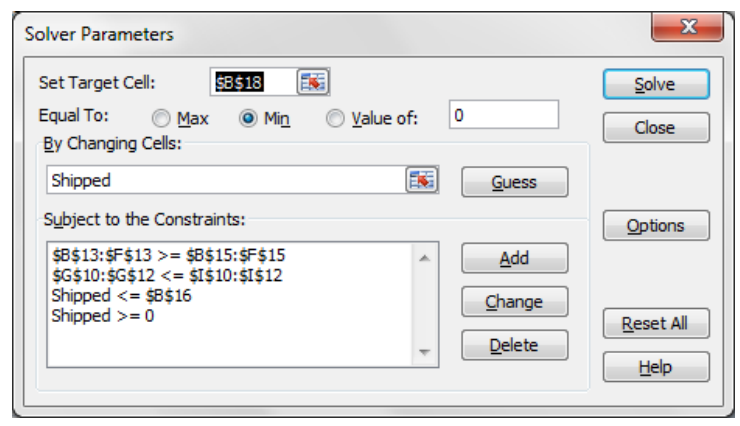

Figure 1. Solver Parameter

Minimized total cost of 2,790 is shown in cell B18 in table 2 . 
Table 3. Answer Report

\begin{tabular}{|c|c|c|c|c|c|c|}
\hline Cell & \multicolumn{2}{|l|}{ Target } & & & & \\
\hline \multirow[t]{2}{*}{ \$\$18 } & Total Cost $>=$ & $\$ 2,790.00$ & & & & \\
\hline & \multicolumn{2}{|l|}{ Adjustable } & \multicolumn{2}{|c|}{ Lower Target } & \multicolumn{2}{|c|}{ Upper Target } \\
\hline Cell & Name & Value & Limit & Result & Limit & Result \\
\hline$\$ B \$ 10$ & Factory 1 Shop 1 & 0 & 0 & 2790 & 0 & 2790 \\
\hline$\$ C \$ 10$ & Factory 1 Shop 2 & 0 & 0 & 2790 & 0 & 2790 \\
\hline$\$ D \$ 10$ & Factory 1 Shop 3 & 0 & 0 & 2790 & 0 & 2790 \\
\hline$\$ E \$ 10$ & Factory 1 Shop 4 & 9000 & 9000 & 2790 & 9000 & 2790 \\
\hline$\$ F \$ 10$ & Factory 1 Shop 5 & 1000 & 1000 & 2790 & 1000 & 2790 \\
\hline$\$ B \$ 11$ & Factory 2 Shop 1 & 0 & 0 & 2790 & 0 & 2790 \\
\hline$\$ C \$ 11$ & Factory 2 Shop 2 & 0 & 0 & 2790 & 0 & 2790 \\
\hline \$D\$11 & Factory 2 Shop 3 & 10000 & 10000 & 2790 & 10000 & 2790 \\
\hline \$E\$11 & Factory 2 Shop 4 & 0 & 0 & 2790 & 0 & 2790 \\
\hline$\$ F \$ 11$ & Factory 2 Shop 5 & 10000 & 10000 & 2790 & 10000 & 2790 \\
\hline$\$ B \$ 12$ & Factory 3Shop 1 & 3000 & 3000 & 2790 & 10000 & 3140 \\
\hline$\$ C \$ 12$ & Factory 3 Shop 2 & 9000 & 9000 & 2790 & 10000 & 2850 \\
\hline \$\$12 & Factory 3 Shop 3 & 2000 & 2000 & 2790 & 10000 & 3350 \\
\hline$\$ E \$ 12$ & Factory 3 Shop 4 & 1000 & 1000 & 2790 & 10000 & 3780 \\
\hline$\$ \$ 12$ & Factory 3 Shop 5 & 0 & 0 & 2790 & 10000 & 4390 \\
\hline
\end{tabular}

In below tables are shown Answer, Sensitivity and Limits report as output of the Microsoft IP Solver.

Table 4. Limits Report

\begin{tabular}{|c|c|c|}
\hline \multicolumn{3}{|l|}{ Target Cell (Min) } \\
\hline Name & Original Value & Final Value \\
\hline \$B\$18 Total Cost >= & $2,790.00$ & $2,790.00$ \\
\hline \multicolumn{3}{|l|}{ Adjustable Cells } \\
\hline Name & Original Value & Final Value \\
\hline \$B\$10 Factory 1 Shop 1 & 0 & 0 \\
\hline$\$ C \$ 10$ Factory 1 Shop 2 & 0 & 0 \\
\hline \$D\$10 Factory 1 Shop 3 & 0 & 0 \\
\hline \$E\$10 Factory 1 Shop 4 & 9000 & 9000 \\
\hline \$F\$10 Factory 1 Shop 5 & 1000 & 1000 \\
\hline \$B\$11 Factory 2 Shop 1 & 0 & 0 \\
\hline$\$ C \$ 11$ Factory 2 Shop 2 & 0 & 0 \\
\hline \$D\$11 Factory 2 Shop 3 & 10000 & 10000 \\
\hline \$E\$11 Factory 2 Shop 4 & 0 & 0 \\
\hline \$F\$11 Factory 2 Shop 5 & 10000 & 10000 \\
\hline \$B\$12 Factory 3 Shop 1 & 3000 & 3000 \\
\hline \$C\$12 Factory 3 Shop 2 & 9000 & 9000 \\
\hline \$D\$12 Factory 3 Shop 3 & 2000 & 2000 \\
\hline \$E\$12 Factory 3 Shop 4 & 1000 & 1000 \\
\hline \$F\$12 Factory 3 Shop 5 & 0 & 0 \\
\hline
\end{tabular}


Table 5. Sensitivity Report

\begin{tabular}{|c|c|c|c|c|c|c|}
\hline \multicolumn{7}{|c|}{ Adjustable Cells } \\
\hline Cell & Name & $\begin{array}{l}\text { Final } \\
\text { Value }\end{array}$ & $\begin{array}{c}\text { Reduced } \\
\text { Cost }\end{array}$ & $\begin{array}{l}\text { Objective } \\
\text { Coefficient }\end{array}$ & $\begin{array}{l}\text { Allowable } \\
\text { Increase }\end{array}$ & $\begin{array}{l}\text { Allowable } \\
\text { Decrease }\end{array}$ \\
\hline$\$ B \$ 10$ & Factory 1 Shop 1 & 0 & 0.12 & 0.1 & $1 E+30$ & 0.12 \\
\hline$\$ C \$ 10$ & Factory 1 Shop 2 & 0 & 0.08 & 0.07 & $1 \mathrm{E}+30$ & 0.08 \\
\hline \$D\$10 & Factory 1 Shop 3 & 0 & 0.08 & 0.08 & $1 \mathrm{E}+30$ & 0.08 \\
\hline$\$ E \$ 10$ & Factory 1 Shop 4 & 9000 & 0 & 0.04 & 0.03 & 0 \\
\hline$\$ F \$ 10$ & Factory 1 Shop 5 & 1000 & 0 & 0.09 & 0 & 0.03 \\
\hline$\$ B \$ 11$ & Factory 2 Shop 1 & 0 & 0.04 & 0.06 & $1 \mathrm{E}+30$ & 0.04 \\
\hline$\$ C \$ 11$ & Factory 2 Shop 2 & 0 & 0.02 & 0.05 & $1 \mathrm{E}+30$ & 0.02 \\
\hline$\$ D \$ 11$ & Factory 2 Shop 3 & 10000 & 0 & 0.04 & 0.02 & 0.03 \\
\hline$\$ E \$ 11$ & Factory 2 Shop 4 & 0 & 0.02 & 0.1 & $1 \mathrm{E}+30$ & 0.02 \\
\hline$\$ F \$ 11$ & Factory 2 Shop 5 & 10000 & -0.03 & 0.1 & 0.03 & $1 \mathrm{E}+30$ \\
\hline$\$ B \$ 12$ & Factory 3 Shop 1 & 3000 & 0 & 0.05 & 0.04 & 0.05 \\
\hline$\$ C \$ 12$ & Factory 3 Shop 2 & 9000 & 0 & 0.06 & 0.02 & 0.06 \\
\hline$\$ D \$ 12$ & Factory 3 Shop 3 & 2000 & 0 & 0.07 & 0.03 & 0.02 \\
\hline$\$ E \$ 12$ & Factory 3 Shop 4 & 1000 & 0 & 0.11 & 0 & 0.03 \\
\hline$\$ F \$ 12$ & Factory 3 Shop 5 & 0 & 0 & 0.16 & $1 \mathrm{E}+30$ & 0 \\
\hline \multicolumn{7}{|c|}{ Constraints } \\
\hline Cell & Name & $\begin{array}{l}\text { Final } \\
\text { Value }\end{array}$ & $\begin{array}{c}\text { Shadow } \\
\text { Price }\end{array}$ & $\begin{array}{l}\text { Constraint } \\
\text { R.H. Side }\end{array}$ & $\begin{array}{c}\text { Allowable } \\
\text { Increase }\end{array}$ & $\begin{array}{c}\text { Allowable } \\
\text { Decrease }\end{array}$ \\
\hline$\$ G \$ 10$ & Factory 1 Sent & 10000 & -0.07 & 10000 & 1000 & 9000 \\
\hline \$ $\$ 11$ & Factory 2 Sent & 20000 & -0.03 & 20000 & 0 & 8000 \\
\hline$\$ G \$ 12$ & Factory 3 Sent & 15000 & 0 & 40000 & $1 \mathrm{E}+30$ & 25000 \\
\hline$\$ B \$ 13$ & Received Shop 1 & 3000 & 0.05 & 3000 & 7000 & 3000 \\
\hline$\$ C \$ 13$ & Received Shop 2 & 9000 & 0.06 & 9000 & 1000 & 9000 \\
\hline$\$ D \$ 13$ & Received Shop 3 & 12000 & 0.07 & 12000 & 8000 & 2000 \\
\hline$\$ E \$ 13$ & Received Shop 4 & 10000 & 0.11 & 10000 & 9000 & 1000 \\
\hline$\$ F \$ 13$ & Received Shop 5 & 11000 & 0.16 & 11000 & 9000 & 1000 \\
\hline
\end{tabular}

With Solver Foundation is easier to make and solve real optimization models. Solver Foundation uses an optimization modeling language (OML) for specifying optimization models; a .NET API and runtime (Solver Foundation Services) for model creation, reporting, analysis and strong built-in solvers [7]. Key features contain:

Modeling and solving action plans by using constraints, goals and data.

Programming in OML, in C\# imperatively, in F\# functionally, or in any .NET language.

Interfaces to known tools such as Excel and SharePoint to create and solve models [7].

Microsoft Solver Foundation is a .NET solution for mathematical optimization and modeling [7].

Below table shows all required data entered in datasheet.

Table 6. First page of the Foundation Solver

\begin{tabular}{|c|c|c|c|c|c|c|c|c|c|}
\hline Source & Destination & SRC & DST & Cost & Shipments & Source & SCR & Sent & Capacity \\
\hline Factory 1 & Shop 1 & 0 & 0 & 0.1 & 0 & Factory 1 & 0 & 10000 & 10000 \\
\hline Factory 1 & Shop 2 & 0 & 1 & 0.07 & 0 & Factory 2 & 1 & 20000 & 20000 \\
\hline Factory 1 & Shop 3 & 0 & 2 & 0.08 & 0 & Factory 3 & 2 & 15000 & 40000 \\
\hline Factory 1 & Shop 4 & 0 & 3 & 0.04 & 10000 & & & & \\
\hline Factory 1 & Shop 5 & 0 & 4 & 0.09 & 0 & Destination & DST & Received & Demand \\
\hline Factory 2 & Shop 1 & 1 & 0 & 0.06 & 0 & Shop 1 & 0 & 3000 & 3000 \\
\hline Factory 2 & Shop 2 & 1 & 1 & 0.05 & 0 & Shop 2 & 1 & 9000 & 9000 \\
\hline Factory 2 & Shop 3 & 1 & 2 & 0.04 & 9000 & Shop 3 & 2 & 12000 & 12000 \\
\hline Factory 2 & Shop 4 & 1 & 3 & 0.1 & 0 & Shop 4 & 3 & 10000 & 10000 \\
\hline Factory 2 & Shop 5 & 1 & 4 & 0.1 & 11000 & Shop 5 & 4 & 11000 & 11000 \\
\hline Factory 3 & Shop 1 & 2 & 0 & 0.05 & 3000 & & & & \\
\hline Factory 3 & Shop 2 & 2 & 1 & 0.06 & 9000 & & & & \\
\hline Factory 3 & Shop 3 & 2 & 2 & 0.07 & 3000 & & & & \\
\hline Factory 3 & Shop 4 & 2 & 3 & 0.11 & 0 & & & & \\
\hline Factory 3 & Shop 5 & 2 & 4 & 0.16 & 0 & & & & \\
\hline
\end{tabular}


Solver Foundation OML with eight (8) constrains is shown below:

Model[

Parameters[

Sets[Any],

SRC,

DST

],

Parameters[

Reals[-Infinity, Infinity],

Cost[SRC, DST]

],

Decisions[

Reals[0, Infinity],

Shipments[SRC, DST]

],

Constraints[

Constraint 1 ->

Shipments[0,0]+Shipments[0,1]+Shipments[0,2]+Shipments[0,3]+Shipments $[0,4]<=10000$,

Constraint2 ->

Shipments[1,0]+Shipments[1,1]+Shipments[1,2]+Shipments[1,3]+Shipments[1,4]<=20000,

Constraint 3 ->

Shipments[2,0]+Shipments[2,1]+Shipments[2,2]+Shipments[2,3]+Shipments[2,4]<=40000,

Constraint4 -> Shipments[0,0]+Shipments[1,0]+Shipments[2,0]>=3000,

Constraint5 -> Shipments[0,1]+Shipments[1,1]+Shipments[2,1]>=9000,

Constraint6 -> Shipments[0,2]+Shipments[1,2]+Shipments[2,2]>=12000,

Constraint7 -> Shipments[0,3]+Shipments[1,3]+Shipments[2,3]>=10000,

Constraint8 -> Shipments[0,4]+Shipments[1,4]+Shipments[2,4]>=11000

]

Goals[

Minimize[

TotalCost -> Annotation[Sum[ $\left.[\mathrm{i}, \operatorname{SRC}\},\{\mathrm{j}, \operatorname{DST}\}, \operatorname{Cost}[\mathrm{i}, \mathrm{j}]{ }^{*} \operatorname{Shipments}[\mathrm{i}, \mathrm{j}]\right]$, "order", 0$]$

]

]] 
Solver Foundation OML with two (2) constrains is shown below:

Model[

Parameters[

Sets[Any],

SRC,

DST

],

Parameters[

Reals[-Infinity, Infinity],

Cost[SRC, DST],

Capacity[SRC],

Demand[DST]

] ,

Decisions [

Reals[0, Infinity],

Shipments[SRC, DST]

],

Constraints[

SentCapacity -> Foreach $[\{x, S R C\}, \operatorname{Sum}[\{z, D S T\}$, Shipments $[x, z]]<=$ Capacity $[x]]$,

ReceivedDemand -> Foreach[\{y,DST $\}, \operatorname{Sum}[\{z, \operatorname{SRC}\}$, Shipments[z,y]]>=Demand[y]]

],

Goals[

Minimize[

TotalCost -> Annotation[Sum[ $[\mathrm{i}, \operatorname{SRC}\},\{\mathrm{j}, \operatorname{DST}\}, \operatorname{Cost}[\mathrm{i}, \mathrm{j}] * \operatorname{Shipments}[\mathrm{i}, \mathrm{j}]]$, "order", 0] ]

]

]

Same solver foundation results will be for both models. 
Table 7. Solver Foundation Results

\begin{tabular}{|c|c|}
\hline \multicolumn{2}{|c|}{ Solver Foundation Results } \\
\hline Name & Value \\
\hline Solution Type & Optimal \\
\hline TotalCost & 2760 \\
\hline Shipments [Factory 1, Shop 1] & 0 \\
\hline Shipments [Factory 1, Shop 2] & 0 \\
\hline Shipments [Factory 1, Shop 3] & 0 \\
\hline Shipments [Factory 1, Shop 4] & 10000 \\
\hline Shipments [Factory 1, Shop 5] & 0 \\
\hline Shipments [Factory 2, Shop 1] & 0 \\
\hline Shipments [Factory 2, Shop 2] & 0 \\
\hline Shipments [Factory 2, Shop 3] & 9000 \\
\hline Shipments [Factory 2, Shop 4] & 0 \\
\hline Shipments [Factory 2, Shop 5] & 11000 \\
\hline Shipments [Factory 3, Shop 1] & 3000 \\
\hline Shipments [Factory 3, Shop 2] & 9000 \\
\hline Shipments [Factory 3, Shop 3] & 3000 \\
\hline Shipments [Factory 3, Shop 4] & 0 \\
\hline Shipments [Factory 3, Shop 5] & 0 \\
\hline & \\
\hline
\end{tabular}

\section{Comparison Between Minimal and Maximal Total Cost}

Below chart shows comparison between minimal and maximal cost for IP Solver respective Microsoft Foundation Solver. Maximal cost for IP Solver is around $60 \%$ higher than minimal cost. Maximal cost for Microsoft Foundation Solver is around $68 \%$ higher than minimal cost. Using optimal distribution network will impact cost and time saving.

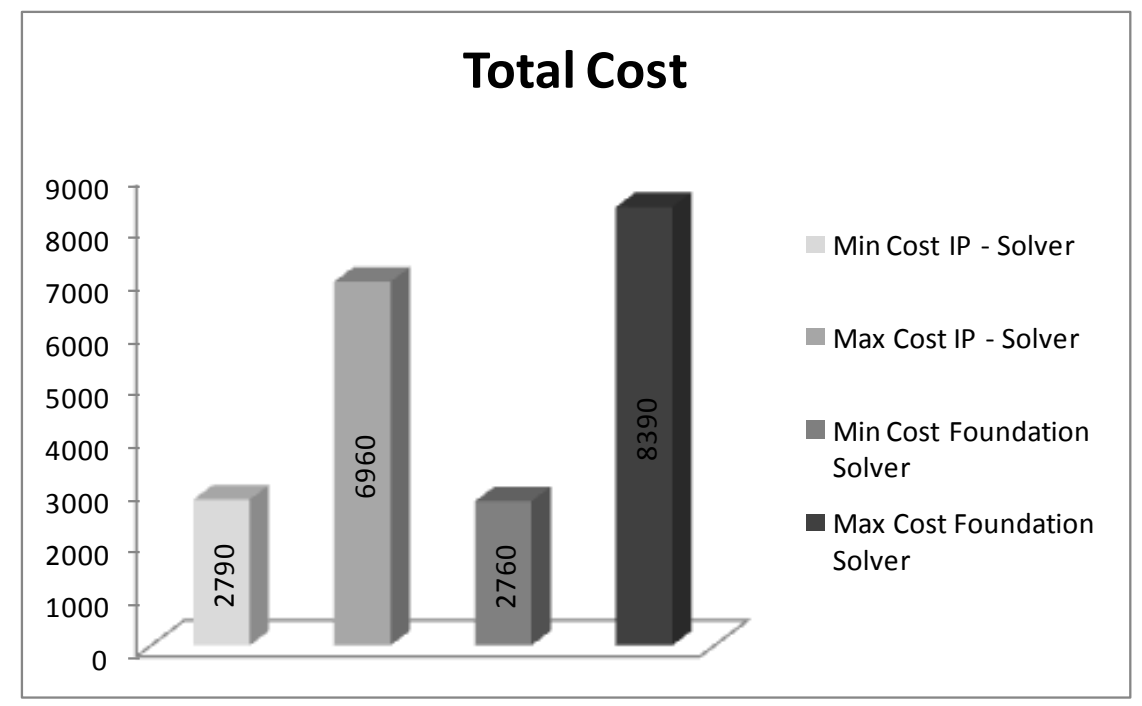

Figure 3. Comparison between min and max cost

Also difference between minimal cost for IP - Solver and Microsoft Foundation Solver is very small, around $1 \%$ while difference between maximal costs is around $17 \%$ as shown in the chart. 


\section{Conclusion}

Logistical route network is flexible and adaptive based on the circumstances and demands. Optimization problems in many fields can be modeled and solved using IP Solver or Microsoft Foundation Solver. In this paper work we have used them to resolve logistical route optimization problem in order to reduce transportation cost. Matrices range in this paper work in $3 \times 5$ but the same procedure will be used for the cases with higher range of the matrices $\mathrm{N} \mathrm{x} \mathrm{M}$.

Optimization problems in general are real world problems we meet in many areas such as mathematics, science, engineering, business and finances. In this matter, we find the optimal or most efficient way of using limited resources to reach objective of the situation. In this paperwork the main goal is maximizing the profit, minimizing the cost, minimizing the total distance travelled and using IP Solver or Microsoft Foundation Solver, this can be achieved very well.

\section{References}

[1] Andrew J. Mason Solving Linear Programs using Microsoft EXCEL Solver

[2] Edwin K.P. Chong and Statislaw H. Zak, An introduction to Optimization, Third Edition

[3] Leslie Chandrakantha Using Excel Solver in Optimization Problems

[4] L. N. Spasovic1, M. P. Boile2 and A.K. Bladikas3, “A Methodological Framework for Optimizing Bus Transit Service Coverage”, 5-8. August 1, 1993

[5] Ronald E. Miller Optimization - Foundation and Application 